\title{
Agresión en adolescentes y su relación con cogniciones, emociones y videojuegos violentos
}

\section{Adolescents' aggression and its relationship to cognitions, emotions and violent videogames}

\author{
Manuel Alfonso Lemos Mosquera*, Pablo Espinosa* \\ *Universidad de A Coruña
}

\begin{abstract}
Resumen
El presente trabajo explora las relaciones entre el consumo de videojuegos y la conducta agresiva, así como el papel mediador de las emociones autoconscientes y las estrategias de desconexión moral. Los resultados muestran relación entre los contenidos violentos de los videojuegos y la violencia manifestada, aunque mediado por las emociones autoconscientes y la desconexión moral. Se comprueba también que el modelo que predice la influencia de los videojuegos en la conducta agresiva se cumple tanto en jóvenes sin problemas de conducta, como en menores en riesgo que manifiestan niveles elevados de conducta antisocial. Palabras clave: Videojuegos, agresión, adolescentes, desconexión moral, emociones
\end{abstract} autoconscientes.

\begin{abstract}
This research explores the relationships between the use of videogames and the agressive behaviour, as well as the mediating role of the self-concious emotions and the moral disengagement. The findings shows the relationship between the violent contents of the videogames and the violence showed, but mediated by the self-concious emotions and the moral disengagement. Its evidenced too that the model that predicts the influence in the aggressive behaviour, its is fulfilled in both youngs without behaviour problems so youngs at risk that shows high levels of antisocial behaviour.

Keywords: Videogames, aggression, adolescents, moral disengagement, selfconcious emotions.
\end{abstract}

Existen una gran cantidad de estudios que describen el efecto de los videojuegos en los jóvenes y adolescentes, centrados en la relación entre el consumo de videojuegos y conductas antisociales, incremento de agresividad y falta de comportamiento prosocial (Anderson, 2004; Anderson \& Bushman, 2001; Anderson et al., 2010).

El Modelo General de Agresión (GAM; Anderson, 2004; Anderson \& Dill, 2000; Anderson \& Bushman 2002; Bushman \& Anderson 2001) considera que son determinadas estructuras de conocimiento las que guían la percepción, la toma de decisiones y la acción de los sujetos y que esas estructuras son aprendidas. El efecto negativo sobre la conducta del consumo excesivo de videojuegos, se explicaría por un proceso de aprendizaje que dichos videojuegos provocan sobre sus usuarios. Además, producen un efecto de facilitación de las conductas agresivas a través de la observación de estas junto con la desensibilización de los procesos emocionales asociados a dichas conductas (Huesman, 2007).

Sin embargo, Ferguson $(2007,2011)$, critica que los estudios realizados hasta el momento no han tenido en cuenta el efecto de terceras variables. Además cuestiona la capacidad de generalización que puedan tener dichos estudios debido a la utilización de muestras de población general, y plantea la necesidad de estudiar poblaciones que manifiesten comportamientos claramente antisociales y agresivos.

La Hipótesis del Desplazamiento Temporal. La mayor parte de los estudios que se han llevado a cabo, han tenido en cuenta los contenidos violentos, sin atender al tiempo de uso de manera independiente (Anderson et al., 2010). Sin embargo, la hipótesis del desplazamiento temporal (Anderson, Huston, Schmitt, Linebarger \& Wright, 2001) defiende que el tiempo excesivo de uso de videojuegos limita el tiempo dedicado a otras actividades sociales, lo cual puede tener un impacto tanto en las habilidades sociales del joven, así como en su desarrollo cognitivo. Lemmens, Valkenburg, y Peter (2011) encuentran que el exceso de uso de videojuegos causa un deterioro de las relaciones sociales existentes. Además, encuentra una relación entre tiempo de juego, incompetencia social y comportamiento agresivo (Lemmens, Valkenburg \& Peter, 2009)

Mediación de Terceras Variables. Las estrategias de desconexión moral se han estudiado previamente como mediadores de la relación entre la agresión y el uso de videojuegos (Richmond \& Wilson, 2008).

Los mecanismos de desconexión moral son estrategias que el individuo utiliza para reestructurar cognitivamente comportamientos moralmente inaceptables con el fin de reducir el afecto negativo 
resultante de haber cometido una transgresión (Bandura, Barbarnelli, Caprara \& Pastorelli, 1996).

Las personas que habitualmente usan este tipo de estrategias, suelen manifestar pocas conductas prosociales y pocos sentimientos de culpa al cometer actos agresivos (Bandura, Caprara, Barbaranelli, Pastorelli, \& Regalia, 2001). Por otro lado, Klimmt, Schmid, Nosper, Hartmann, \& Vorderer, (2006) argumentan que los jugadores de videojuegos violentos necesitan emplear este tipo de mecanismos a fin de justificar los actos cometidos en el juego.

Se ha relacionado, además, el efecto que las emociones autoconscientes, principalmente la culpa y la vergüenza, tienen en la regulación del comportamiento agresivo y antisocial (Tangney \& Dearing, 2002).. Puesto que el sentimiento de culpa es un inhibidor de la conducta antisocial (Olthof, 2012), es posible que los mecanismos de desconexión moral estén jugando un papel fundamental a la hora de evitar tal sentimiento.

\section{Objetivos e hipótesis}

El objetivo del estudio es desarrollar un modelo explicativo de la relación entre videojuegos y violencia a través de variables cognitivas como la desconexión moral y afectivas como la culpa, comparando adolescentes con y sin problemas de conducta.

En concreto se plantearon las siguientes hipótesis:

Hipótesis 1. La atracción por los contenidos violentos en los videojuegos predice un aumento de el comportamiento agresivo.

Con respecto a la hipótesis del desplazamiento temporal se plantea la siguiente hipótesis:

Hipótesis 2. El tiempo de juego, independientemente de los contenidos violentos, predice un aumento de las conductas agresivas.

En cuanto al papel de las variables mediadoras, se plantea lo siguiente:

Hipótesis 3. Las emociones autoconscientes predicen un menor nivel de conducta violenta.

Hipótesis 4. El empleo de estrategias de desconexión moral predice la conducta violenta.

Hipótesis 5. El empleo de videojuegos violentos se relaciona negativamente con las emociones autoconscientes percibidas.

Hipótesis 6. El empleo de videojuegos violentos predice un nivel elevado en el uso e de estrategias de desconexión moral.

Hipótesis 7. Existirá un efecto de mediación de variables cognitivas y afectivas sobre la relación entre el uso de videojuegos y la agresión.

\section{Método}

\section{Participantes}

En el estudio participaron un total de 176 adolescentes españoles de ambos sexos, con edades entre 14 y 20 años (edad media 16,12 desviación típica $=1,277)$. De estos, 111 (59 chicos y 52 chicas) eran alumnos de instituto. Los restantes 65 participantes ( 45 chicos y 20 chicas) eran menores en situación de riesgo social, en diferentes condiciones de cumplimiento o de prevención de medidas penales o de protección.

\section{Instrumentos}

Los participantes respondieron a los siguientes cuestionarios: Cuestionario de Consumo de videojuegos, con preguntas relativas al tiempo y frecuencia de uso, así como a sus contenidos, violentos o no; el Cuestionario de Desconexión Moral (Bandura, Barbarenelli, Caprara \& Pastorelli, 1996); el Test of Self-Conscious Affect (TOSCA-3) (Tangney \& Dearing, 2002). Inventario de Comportamientos Antisociales (Espinosa \& Clemente, 2011).

\section{Procedimiento}

Se solicitó el consentimiento de los menores y los cuestionarios se aplicaron de forma colectiva, bien en el aula (alumnos de secundaria), bien en salas de uso común de centros sociales (menores en riesgo). Tras la finalización del estudio, se proporcionó información a los profesores/tutores y a los participantes sobre las características y los objetivos del estudio.

\section{Análisis de datos}

Se realizaron análisis descriptivos y pruebas $t$ para comprobar las diferencias entre sexos y entre los grupos de menores con y sin problemas de conducta. Se llevó a cabo un análisis correlacional para determinar la hipótesis sobre el desplazamiento temporal y también un análisis de mediación a través de un modelo de ecuaciones estructurales.

\section{Resultados}

\section{Análisis de fiabilidad}

Las escalas aplicadas mostraron los siguientes índices de fiabilidad en nuestra muestra: Violencia Directa $\alpha=.88$ Desconexión moral $\alpha=.87$ y Culpa $\alpha=.74$

\section{Análisis descriptivo y comparaciones entre grupos y sexos}

Los participantes mostraron puntuaciones moderadas en cuanto a la afición por los juegos violentos $(M=3,5$; $D T=2,0)$ así así como en su uso $(M=2,34 ; D T=1,4)$. La frecuencia de juego fue bastante baja $(M=1,96 ; D T=$ 1,2)

Se observaron diferencias en función del sexo. Las mujeres manifestaron una preferencia claramente menor por los videojuegos $(t(174)=7.75 \mathrm{p}<.001$; Varones: $M=4.75 ; D T=1,61$ vs. Mujeres: $M=2.74 ; D T=1,80)$, así como por la necesidad de contenidos violentos para que estos sean divertidos $(t(174)=6,04 \quad \mathrm{p}<.001$; Varones: $M=4.17 ; D T=1,63$ vs. Mujeres: $M=2.74 ; D T=1,43)$, y también muestraron un menor tiempo de utilización $(t(174)=4,11 \mathrm{p}<.001$; Varones: $M=2,63 ; D T=1,12 \mathrm{vs}$. Mujeres: $M=1,92 ; D T=1,14)$. En cuanto a las variables cognitivas y de agresión, encontramos en todas ellas diferencias significativas por sexos. Las mujeres mostraron una mayor tendencia a la culpa cuando comenten una transgresión $(t(174)=3,59 \quad \mathrm{p}<.001$; Varones: $M=3,58 ; D T=, 62$ vs. Mujeres: $M=3,91$; $D T=, 68)$ y a usar menos mecanismos de desconexión moral $(t(174)=3,15 \mathrm{p}<.01$; Varones: $M=2,98 ; D T=, 82$ vs. Mujeres: $M=2,59 ; D T=, 75)$. Por último, las mujeres 
comentían significativamente menos agresiones que los varones $(t(174)=2,57 \mathrm{p}<.05$; Varones: $M=1,60 ; D T=$ ,50 vs. Mujeres: $M=1,42 ; D T=, 39)$.

Con respecto a las diferencias entre grupos en el consumo de videojuegos, sólo encontramos que los menores sin problemas utilizaban más videojuegos que los menores en riesgo, $(t(174)=2,68 \quad \mathrm{p}<.01$; Menores sin problemas: $M=2,51 ; D T=1,13$ vs. Menores en riesgo: $M=2,03 ; D T=1,18)$. No existían diferencias significativas entre grupos en la atracción por la violencia en los videojuegos. Sin embargo, los menores en riesgo tienían menos tendencia a la culpa $(t(174)=$ $3,22 \mathrm{p}<.01$; Menores sin problemas: $M=3,83 ; D T=, 53$ vs. Menores en riesgo: $M=3,51 ; D T=, 74)$, utilizaban más los mecanismos de desconexión moral $(t(174)=$ $2,72 \mathrm{p}<.01$; Menores sin problemas: $M=2,69 ; D T=, 69$ vs. Menores en riesgo: $M=3,03 ; D T=, 96)$ y eran más agresivos $(t(174)=5,75 \mathrm{p}<.001$; Menores sin problemas: $M=1,39 ; D T=, 31$ vs. Menores en riesgo: $M=1,77$; $D T=, 58)$.

\section{Análisis de correlación parcial}

La correlación parcial entre tiempo de uso de los videojuegos y el comportamiento violento, controlando por la atracción por los contenidos violentos no fue significativa $(\mathrm{r}=.09, \mathrm{p}=\mathrm{ns})$, lo que indica que para nuestros participantes el tiempo de uso por si sólo no predice el comportamiento antisocial.

\section{Análisis de mediacion}

El modelo mostró que para ambos grupos, el efecto total del uso de videojuegos violentos sobre el comportamiento agresivo fue significativo, mientras que el efecto directo mientras que el efecto directo no lo fue cuando introdujimos como mediadores la desconexión moral y las emociones morales (culpa). El modelo global mostró un ajuste aceptable y los efectos directos entre predictor $\mathrm{y}$ mediadores $\mathrm{y}$ entre mediadores $\mathrm{y}$ criterio son significativos en todos los casos, completándose el criterio de mediación. Estos efectos persisten cuando controlamos por la variable sexo.

\section{Menores en riesgo}

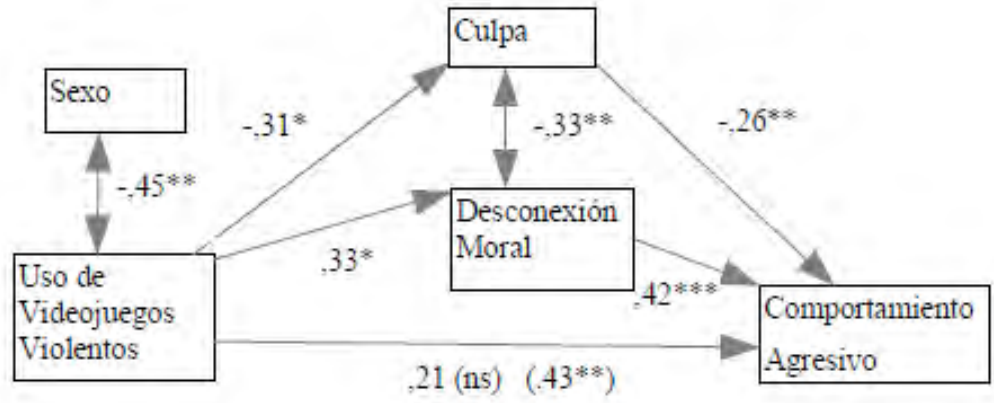

Menores sin problemas de comportamiento

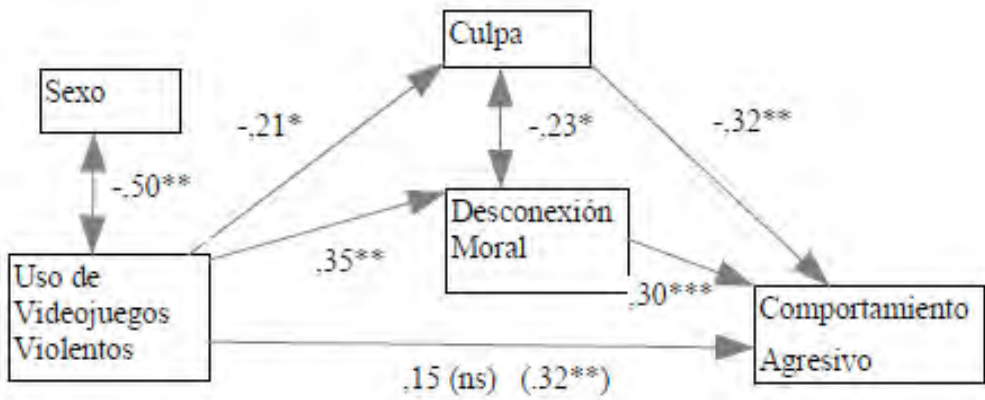

* $\mathrm{p}<0.05 ; * * \mathrm{p}<0.01 ; * * * \mathrm{p}<0.001 ;$ ns no significativo. los valores entre paréntesis son efectos totales

$\chi 2(6,176)=10,692 \mathrm{p}=.098 ; \mathrm{RMSEA}=.067 ; \mathrm{NFI}=.943 ; \mathrm{CFI}=972$

Figura 1. Efectos directos en el modelo de mediación de la desconexión moral y la culpa sobre la relación entre videojuegos y agresión

Por otro lado, los ratios críticos para las diferencias entre parámetros no muestran diferencias significativas entre ambos grupos, de modo que las relaciones entre variables se mantienen de un grupo a otro.

\section{Discusión y conclusiones}

La principal aportación de este estudio, a pesar de no haber logrado acceder a una muestra extensa, es haber podido verificar las relaciones entre las variables del 
estudio tanto en menores sin problemas de conducta como en menores en riesgo, ya que los menores con problemas de conducta constituyen una población de difícil acceso y de la que no se pueden conseguir datos de forma sencilla. Si bien en lo que respecta a estos dos grupos de menores no existía ninguna hipótesis previa sobre posibles efectos diferenciales de las variables del modelo, investigaciones previas (Ferguson 2007, 2011) ponían de manifiesto la importancia de contar con este tipo de muestras para superar las limitaciones de estudios previos. Nuestros resultados han confirmado las hipótesis que planteábamos para el estudio, con excepción de la hipótesis segunda, que trataba de verificar la hipótesis del desplazamiento temporal.

Tabla 1.

Correlaciones bivariadas para las variables en el modelo de mediación.

\begin{tabular}{|c|c|c|c|c|c|}
\hline & $\begin{array}{l}\text { Videojuegos } \\
\text { Violentos }\end{array}$ & Violencia Directa & $\begin{array}{l}\text { Desconexión } \\
\text { Moral }\end{array}$ & Culpa & Sexo \\
\hline Videojuegos Violentos & & $32 * *$ & $.35^{* *}$ & $-.21 *$ & -.50 \\
\hline Violencia Directa & $.43 * *$ & & $.44^{* *}$ & $-.44 * *$ & $-.28 * *$ \\
\hline DesconexiónMoral & $.38 * *$ & $59 * *$ & & $-.28 * *$ & $-.34 * *$ \\
\hline Culpa & $-.31 *$ & $-.49 * *$ & $-.39 * *$ & & $.27 * *$ \\
\hline Sexo & $-.45 * *$ & -.09 & -.08 & .23 & \\
\hline
\end{tabular}

Los resultados muestran un efecto total significativo de los contenidos violentos sobre el comportamiento agresivo en ambos grupos, de acuerdo con lo que se planteaba en la primera hipótesis

En la segunda hipótesis pretendíamos comprobar el efecto que el tiempo de uso de los videojuegos producía sobre la conducta violenta. No obstante parece que, y hasta donde alcanzan nuestro resultados, ese efecto no se produce. Así, no se ha encontrado apoyo para las explicaciones basadas en la hipótesis del desplazamiento temporal (Lemmens et al., 2011) frente a las explicaciones basadas en los efectos de los contenidos violentos.

Los resultados con respecto a la relación entre desconexión moral y emociones morales con el comportamiento violento muestran apoyo para las hipótesis 3 y 4.

También se cumple la relación entre videojuegos violentos y emociones morales y desconexión moral en el sentido predicho por las hipótesis 5 y 6 .

Finalmente, el modelo de ecuaciones estructurales planteado para explorar los posibles efectos de mediación muestra un buen ajuste y se observa como el efecto directo de los videojuegos violentos sobre la agresión, descontando los efectos indirectos atribuibles a la desconexión moral y la culpa, deja de ser significativo. Esto indica que ambas variables son relevantes para explicar los mecanismos por los que los videojuegos pueden tener un efecto sobre la conducta.

Resulta especialmente interesante poder comentar que el modelo estructural que muestran las variables implicadas en nuestro estudio se cumple tanto en los menores en riesgo como en los que no presentan problemas de conducta. Evidentemente esto no cierra la cuestión, pero sí parece ser una indicación de que los estudios previos realizados en poblaciones normalizadas son generalizables a los sujetos que manifiestan claros niveles de conducta antisocial. Es obvio que es necesario profundizar en esta cuestión con nuevas investigaciones.

Tal y como se predecía desde los enfoques asociados al Modelo General de Agresión (Anderson, 2004; Anderson \& Dill, 2000; Anderson \& Bushman 2002; Bushman \& Anderson 2001), el elemento central que se ha relacionado como determinante del efecto de los videojuegos sobre el comportamiento agresivo, son los contenidos violentos. Sin embargo, no ha de perderse de vista que los resultados obtenidos evidencian que el efecto de los contenidos violentos sobre el comportamiento agresivo, están mediados por el uso de la desconexión moral y el sentimiento de culpa. Es obvio, como ya hemos señalado, que no todos los usuarios de videojuegos cometen transgresiones, y esa diferencia en el efecto final que la exposición a contenidos violentos produce en las personas, ha de ser entendida en base a dichas variables cognitivas y emocionales. No obstante, tener en cuenta las calificaciones de edad de los videojuegos en función del contenido parece una práctica mas que recomendable a la vista de las relaciones entre variables encontradas.

Hemos encontrado relaciones significativas entre terceras variables de tipo cognitivo y emocional, en concreto las estrategias de desconexión moral y la emoción autoconsciente de culpa. La falta de comprobación de este tipo de relaciones era también otra crítica de Ferguson (2007, 2011) a las investigaciones que han estudiado la relación entre uso de videojuegos y violencia.parece que tal y como apuntaban Tangney y Dearing, (2002) el sentimiento de culpa es un freno muy potente de la conducta agresiva, y es necesario inhibirlo de alguna manera a fin de llevar a cabo algún tipo de transgresión (Klimmt et al 2006; Olthof, 2012). La culpa, más que otras emociones morales ante transgresiones como la vergüenza, es relevante en este sentido porque se trata 
de una emoción proactiva que lleva al individuo a modificar su comportamiento futuro tras cometer una transgresión o incluso previamente a cometerla, si el individuo es capaz de anticipar los sentimientos negativos que puede provocar en el realizar un comportamiento antisocial.

Este estudio ha empleado una metodología correlacional, con lo que no es posible establecer relaciones causales entre variables. Asimismo el carácter tranversal del trabajo hace que las generalizaciones que a partir de este puedan realizarse deban tomarse con cautela.

No obstante, si bien la muestra de menores en riesgo es escasa, la dificultad de acceso a este tipo de población, hace que los datos obtenidos de esta muestra sean relevantes.

\section{Referencias}

Anderson, C. A. (2004). An update on the effects of playing violent video games. Journal of Adolescence, 27(1), 113-122. doi:10.1016/ j.adolescence.2003.10.009

Anderson, C. A., \& Bushman, B. J. (2001). Effects of violent video games on aggressive behavior, aggressive cognition, aggressive affect, physiological arousal, and prosocial behavior: A meta-analytic review of the scientific literature. Psychological Science, 12(5), 353-359. doi:10.1111/14679280.00366

Anderson, C. A., \& Bushman, B. J. (2002). Human aggression. Psychology, 53(1), 27.

Anderson, C. A., \& Dill, K. E. (2000). Video games and aggressive thoughts, feelings, and behavior in the laboratory and in life. Journal of Personality and Social Psychology, 78(4), 772.

Anderson, C. A., Shibuya, A., Ihori, N., Swing, E. L., Bushman, B. J., Sakamoto, A., . . Saleem, M. (2010). Violent video game effects on aggression, empathy, and prosocial behavior in eastern and western countries: A meta-analytic review. Psychological Bulletin, 136(2), 151-173. doi:10.1037/a0018251

Anderson, D. R., Huston, A. C., Schmitt, K. L., Linebarger, D. L., \& Wright, J. C. (2001). Early childhood television viewing and adolescent behavior: The recontact study - Introduction. [Review]. Monographs of the Society for Research in Child Development, 66(1), 1-154. doi:10.1111/15405834.00121

Bandura, A., Barbaranelli, C., Caprara, G. V., \& Pastorelli, C. (1996). Mechanisms of moral disengagement in the exercise of moral agency. Journal of Personality and Social Psychology, 71(2), 364.
Bandura, A., Caprara, G. V., Barbaranelli, C., Pastorelli, C., \& Regalia, C. (2001). Sociocognitive self-regulatory mechanisms governing transgressive behavior. Journal of Personality and Social Psychology, 80(1), 125-135. doi:10.1037//00223514.80.1.125

Bushman, B. J., \& Anderson, C. A. (2001). Is it time to pull the plug on hostile versus instrumental aggression dichotomy? Psychological Review, 108(1), 273.

Espinosa, P., \& Clemente, M. (2011). La medida del comportamiento antisocial en adolescentes y jóvenes: Desarrollo del inventario de comportamientos antisociales (ICA). Revista De Psicología Social, 26(2), 223-240. doi:10.1174/021347411795448974

Ferguson, C. J. (2007). Evidence for publication bias in video game violence effects literature: A metaanalytic review. Aggression and Violent Behavior, 12(4), 470-482. doi:10.1016/j.avb.2007.01.001

Ferguson, C. J. (2011). Video games and youth violence: A prospective analysis in adolescents. Journal of Youth and Adolescence, 40(4), 377-391. doi:10.1007/s10964-010-9610-x

Huesmann, L. R. (2007). The impact of electronic media violence: Scientific theory and research. Journal of Adolescent Health, 41(6), S6-S13.

Klimmt, C., Schmid, H., Nosper, A., Hartmann, T., \& Vorderer, P. (2006). How players manage moral concerns to make video game violence enjoyable. Communications, 31(3), 309-328.

Lemmens, J. S., Valkenburg, P. M., \& Peter, J. (2009). Development and Validation of a Game Addiction Scale for Adolescents. Media Psychology, 12(1), 7795. doi:10.1080/15213260802669458

Lemmens, J. S., Valkenburg, P. M. \& Peter, J. (2011). The effects of pathological gaming on aggressive behavior. Journal of Youth and Adolescence, 40(1), 38-47. doi:10.1007/s10964-010-9558-x

Olthof, T. (2012). Anticipated feelings of guilt and shame as predictors of early adolescents' antisocial and prosocial interpersonal behaviour. European Journal of Developmental Psychology, 9(3), 371388. doi:10.1080/17405629.2012.680300

Richmond, J., \& Wilson, J. C. (2008). Are graphic media violence, aggression and moral disengagement related? Journal of Managerial Psychology, 15(2), 350-357. doi:10.1080/13218710802199716

Tangney, J. P., \& Dearing, R. L. (2002). Shame and guilt Guilford Press.

\section{Agradecimientos}

Queremos agradecer la colaboración de la Familia Salesiana, al IES de Pastoriza-Arteixo y a la Fundación Hogar de Santa Margarita. 